\title{
Improving Care for Older Persons with Schizophrenia through an Academic-Community Partnership
}

\author{
Laurie A. Lindamer, PhD, Barry D. Lebowitz, PhD, Richard L. Hough, PhD, Piedad Garcia, \\ Ed.D., LCSW, Alfredo Aquirre, LCSW, Maureen C. Halpain, MS, Colin Depp, PhD, and Dilip V. \\ Jeste, MD
}

To provide quality care for persons with schizophrenia, evidence-based pharmacological and psychosocial research developments must be efficiently translated into community practice. Academic-community partnerships can facilitate this translation $(1 ; 2)$. Using the principles of community-based participatory research (CBPR) (3) and cultural exchange theory (CET) (4) we developed a partnership between an academic research center and a large public mental health system with the goal of improving care for middle-aged and older people with schizophrenia and other psychoses.

Although CBPR is solidly established in many areas of public health research, it is less so in mental health systems $(3 ; 5)$. Featuring a process of shared decision-making, CBPR empowers the community as an equal partner in the research process. Public participation in all phases of research insures clinical and cultural relevance to communities, and it contributes to the effectiveness and sustainability of the interventions, programs, and evidence-based practices (EBPs) that result from such research (6).

CET describes a transaction of knowledge, attitudes, and practices that occurs when two groups representing diverse cultural systems (e.g., ethnic, professional, organizational) interact and engage in a process of debate and compromise (4). The development of the partnership involves cultural exchange, a bi-directional process in which both parties contribute equally, derive benefit and change as a result of the transaction (4).

We describe our use of the principles of CBPR and CET to create a stable and productive research partnership between the San Diego Adult and Older Adult Mental Health Services (AOAMHS) and the Geriatric Psychiatry Research Center (GPRC) of the University of California, San Diego (UCSD).

\section{The Partners}

Although there was a longstanding clinical collaboration between UCSD and the AOAMHS, there was little interaction in terms of research. In 2002, as part of an NIMH grant the UCSD and the AOAMHS began a clinical research partnership.

AOAMHS provides public -supported mental health services for about 40,000 San Diegans over age 18 annually. The county is ethnically diverse- $50 \%$ of AOAMHS users are Caucasian, 19\% Latino, and 5\% African American.

\section{A Collaborative Process Model}

Public-academic partnerships combine two different organizational systems, each with its own values, styles, limitations, and motivators. The Collaborative Process Model we employed to develop the organization and function of the AOAMHS/GPRC partnership (7;8) consists of the following five main steps: (I) Building and sustaining the partnership, (II) Mobilizing 
community support and enhancing infrastructure for community research capacity, (III)

Knowledge generation (research and training), (IV) Knowledge transfer to community practice (dissemination and implementation), and (V) Evaluation of the outcomes and process. Applying the core principles of CBPR and CET to the Collaborative Process Model, we undertook the formation of an academic-public partnership.

\section{Building Community Partnership}

The collaboration began by discussing the goals and objectives of each partner, identifying areas of overlap, and recognizing benefits to each organization. Researchers often find bureaucratic processes in public service organizations cumbersome, and AOAMHS staff reported similar perceptions of the University's bureaucracy (9-11). Several other cultural differences were identified (e.g., differences in decision making styles). To the extent possible, we compromised to meet the objectives of the partnership. In those circumstances where consensus was not possible, we accepted the other's decision and respected the cultural context in which it was made $(3 ; 10)$. Creating feasible and useful joint operations for the partnership required time, trust, and mutual respect $(9 ; 10)$, and consequently, these interactions significantly changed both organizations.

Consistent with CET and CBPR, we began the partnership with a priority-setting process. Both partners presented their mission, goals, and structure. The following initiatives were identified: recruitment into research protocols, needs assessment, utilization analyses, and public education. Below, we describe some of the processes that were important in building the organization and structure of the partnership and in achieving the jointly determined objectives.

\section{Oversight and Representation}

The initial structure of the partnership consisted of three committees with equal representation of UCSD and County: 1) Staff to discuss ongoing operations and projects, 2) Administrative to discuss policy issues and resource allocation, and 3) Executive to discuss overall priorities and progress.

\section{Staffing}

To create cohesion and increase communication, we hired staff specifically for the partnership. Functioning as equal partners in the decision-making process, we hired a community mental health liaison and a data analyst. These joint staff were housed at County facilities and employed by the University, providing an effective way of "blurring the lines" between the County and the GPRC.

\section{Bureaucratic Arrangements}

Several fiscal and administrative problems emerged in forming the partnership. For example, we initially planned for the County to administer the partnership budget through a subcontract with UCSD. The acceptance of external grant funding, however, presented the County with administrative and procedural challenges. The resolution required that each institution look beyond its distinct set of organizational priorities and loyalties. It was decided that UCSD would manage the entire budget and become the designated employer of all staff. The partners, however, retained joint determination of budget allocations, personnel selection, and supervision. This agreement was documented in a Memorandum of Understanding that outlined the terms of the collaboration and provided for annual review and revision.

\section{Administrative Challenges}

One priority of the partnership was to develop mechanisms to enhance recruitment of representative community samples for research. To accomplish this, the partners engaged in 
the bidirectional process posited by the CET. UCSD and the County each had institutional mechanisms to track research projects. The County had limited capacity to review and monitor projects, thus restricting the number of protocols active in county programs. Working collaboratively, the partners standardized several processes to reduce the burden on the organizations and investigators. We jointly created databases to track projects, subject participation, and publications. The efforts of the shared staff facilitated identification of new recruitment sources and reduced the time spent on duplicative administrative aspects. As the result of this endeavor, policies and procedures at both organizations were modified.

\section{Changes in Context}

Public-academic partnerships are established within a fluid context of political processes, changing priorities, and other events that require flexibility and adaptation. For example, during the formation of the partnership the Privacy Rule of the Health Insurance Portability and Accountability Act (HIPAA) was enacted. Complying with HIPAA resulted in a resetting of project timetables, the development of a new Data Use Agreement, and several other procedures to ensure that the data transfer was HIPAA-compliant. That the collaboration survived and flourished in changing context indicates the strength of the partnership and the validity of the pursuit.

\section{(II) Mobilizing Community Support and Enhancing Infrastructure for Research Capacity}

To accomplish the second step of our Collaborative Process Model, we developed several innovative mechanisms designed to involve the community and promote research.

\section{Participation of Community Members}

We formed a Community Advisory Board (CAB) chaired by a community member and composed of investigators, administrators, consumers, caregivers, and care providers. The Committee reviewed every research protocol prior to its submission to the University Institutional Review Board (IRB) and the AOAMHS Research Committee to assess public health significance, feasibility, and adequacy of protection of participants' rights. The CAB also monitored each study's progress. Input from this $\mathrm{CAB}$ assisted in identifying new recruitment opportunities, reducing participant burden, and improving communication between clinicians and researchers.

\section{Technical and Financial Support}

AOAMHS research infrastructure was upgraded by providing ongoing data management and analysis, general methodological and other scientific support for outcomes assessment, and other practice and clinical research activity.

\section{Increasing Community-based Research}

The partnership provided an environment for the design and support of community-based studies and made pilot project funding available to support development activities. Projects from University and County investigators were solicited, and the Community Advisory Board reviewed each proposal.

\section{Enhancing Support}

Both partners participated in increasing community awareness of and support for the collaboration by conducting educational programs throughout the county, and AOAMHS provided opportunities for improving UCSD researchers' understanding of community-related issues. 


\section{Expanding Community Capacity}

The passage of Proposition 63 (Mental Health Services Act), which generates new tax revenue specifically earmarked for mental health services, required that each county assess and prioritize its own mental health needs. The UCSD-AOAMHS partnership was instrumental in conducting and analyzing the needs assessment and service utilization data that formed the core of the San Diego plan, which was approved by the State's review committees.

\section{(III) Knowledge Generation (Research and Training)}

The third step in the Collaborative Process Model is to provide the background and descriptive analyses of the community system, providing a basis for future research and program development.

\section{Needs Assessment Project}

The partners conducted a collaborative qualitative study to assess the mental health needs of older adults in the community. Consistent with CBPR, several community groups participated in the data collection, including the Coalition (see below), NAMI San Diego, and AOAMHS. A town hall meeting was held to share the results with the community and a report was also made available on the University and county websites.

\section{Service Utilization Data Analyses}

Another jointly agreed upon priority of the partnership was the review of AOAMHS service utilization. To accomplish this, AOAMHS transferred their Management Information System (MIS) database to Center researchers in accordance with HIPAA, UCSD IRB, and AOAMHS Research Committee regulations. Although this was a lengthy process, the database has become a unique resource. It contains six years of service utilization data, capturing characteristics and service data for over 40,000 consumers. These analyses have helped the partners gain better understanding of service use and costs of mental health care and to identify potential targets for the development of interventions, and several manuscripts, co-authored by GRPC and AOAMHS partners have been published.

\section{Evaluation of System-Wide Interventions}

Program evaluation is a critical activity for County. The formation of the partnership, in particular the University's data management resources and analytic expertise, allowed the County to examine in more detail the impact of system-wide interventions. For example, when AOAMHS had to eliminate all Day Rehabilitation programs due to budget reductions, we collaborated on a retrospective analysis of mental health service use of clients whose care was transferred to clubhouse programs. Not only did this project provide information that was important for county planning purposes, but it also demonstrated the partnership's ability to evaluate outcomes of a system-wide change in services, an activity that will continue to be valuable to both the County and the University.

\section{System-wide Assessment of Cultural Competence}

To comply with state requirements to evaluate the cultural responsiveness of the AOAMHS care providers, the partners surveyed over 2,000 AOAMHS administrative, clinical, and support staff members. The results of this survey led to county-wide programs to provide culture-fair assessments and culturally sensitive treatments. These findings were published in a report that has been widely disseminated in the county and the state. 


\section{(IV) Knowledge Transfer (Dissemination and Implementation)}

The fourth step in our Center's Collaborative Process Model involves communication of findings, with the goal of changing community practice. Although presenting study findings in scientific publications is a necessary means of dissemination, it is insufficient. Consequently, we have sought to disseminate our research findings to the community in innovative ways that are described below.

\section{Wellness Campaign}

To increase public and professional awareness of the mental health needs of older adults, we jointly developed a major educational program, "The Wellness Campaign", which consisted of a series of lectures by national experts that was held in accessible venues throughout the county.

\section{Newsletter and Websites}

We collaborated on a quarterly publication distributed both electronically and in print that targeted individuals with psychoses, families, professionals, researchers, advocates, and the public.

\section{San Diego Older Adult Mental Health and Substance Abuse Coalition ("Coalition") \\ One of the innovative, collaborative methods of dissemination was the formation of a Coalition of stakeholders that focuses on education and advocacy for the mental health needs of older adults. Recently, the Coalition was adopted as a formal program of NAMI San Diego.}

\section{White House Conference on Aging}

The partners co-sponsored an officially sanctioned "Mini-Conference to the 2005 White House Conference on Aging", which resulted in a "white paper" published in English and in Spanish. This document summarizes the proceedings of the meeting that was attended by over 120 consumers, advocates, and providers of older adult mental health care.

\section{Development and Dissemination of Practice Guidelines}

Using the practice guidelines for the use of antipsychotics and other treatments for older people with schizophrenia (12), the partners are developing programs to disseminate and implement these practice guidelines in the county's mental health clinics.

\section{(V) Evaluation of the Process and the Success of the Partnership}

To accomplish the fifth step in the Center's Process Development Model, we recently surveyed academic and community stakeholders to evaluate the processes and outcomes of the collaboration. Overall, the partnership received high ratings on the quality and the dedication of those involved in the collaboration and on innovative problem solving. Stakeholders also noted that the partnership would benefit from even greater involvement of consumers, caretakers, and clinicians. Respondents stated that the natural tension between priorities of different organizations sometimes resulted in conflicts of interest and challenges in identifying mutually beneficial projects, which may have hindered the partnership's efficiency. Feedback from the evaluation also suggested that a major priority should be to emphasize research that has a direct, observable, and sustainable impact on community practices. In general, most stakeholders agreed that the partnership had successfully evolved from two separate and complex organizations to a joint collaboration with shared goals. 
In response to this feedback, we have made revisions in infrastructure and priorities for the ongoing research partnership. To increase the role of consumers and caregivers in the research planning and oversight processes, we have made the following three major infrastructure changes: 1) developed a Partners' Council with representation of NAMI and frontline clinicians, 2) formed a Consumer Liaison Unit including a Consumer Advisory Board, and 3) increased interactions with local and national mutual support and mental health advocacy organizations.

Beginning with the Collaborative Process Model and modifying it to include the basic principles of CET and CBPR, UCSD and AOAMHS created a partnership focused on improving care for middle-aged and older adults with schizophrenia and other psychoses. Consistent with CET, the interaction between the two organizations that differed in values, bureaucracy, and function required a substantial investment of time, strong commitment to the process, flexibility in the face of shifting priorities, and willingness to compromise and accommodate. This academic-public partnership has been a difficult undertaking, and many of the benefits have yet to come to fruition. Nonetheless, there are some tangible benefits to both partners. AOAMHS has developed an infrastructure to support research, educational programs, and the development of the mental health delivery system for older adults. The University has gained knowledge and awareness of community mental health services conditions and improved its ability to develop and implement effective community-based research projects.

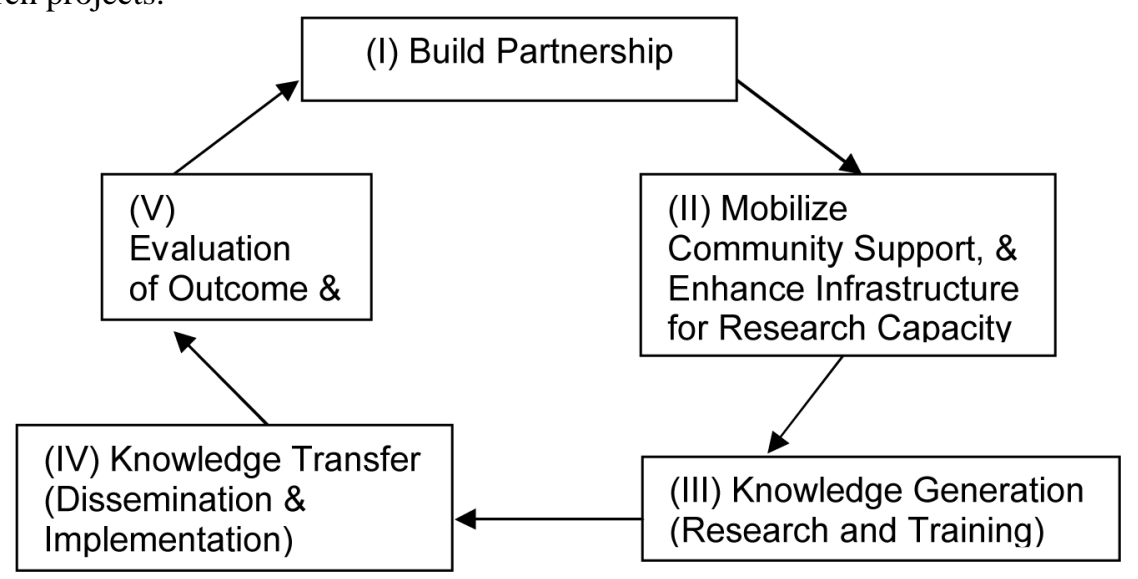

\section{REFERENCES}

1. National Advisory Mental Health Council: Bridging Science and Service. Publication 99-4353 National Institutes of Health; Bethesda, MD: 1999.

2. Zerhouni E. Medicine: The NIH Roadmap. Science 2003;302:63-72. [PubMed: 14526066]

3. Israel BA, Schulz AJ, Parker EA, et al. Review of community-based research: Assessing partnership approaches to improve public health. Annu Rev Public Health 1998;19:173-202. [PubMed: 9611617]

4. Palinkas LA, Allred CA, Landverk J. Models of research-operational collaboration for behavioral health in space. Aviation, Space and Environmental Medicine 2005;76:B52-B60.

5. Wells K, Miranda J, Bruce ML, et al. Bridging community intervention and mental health services research. American Journal of Psychiatry 2004;161:955-963. [PubMed: 15169681]

6. Wells KB, Stauton A, Norris KC, et al. Building an academic-community partnered network for clinical services: the community Health Improvement Collaborative (CHIC). Ethnicity and Disease 2006;16:S1-3-S1-17.

7. Veazie MA, Teufel-Shone NI, Silverman GS, et al. Building community capacity in public health: the role of action-oriented partnerships. J Public Health Manag Pract 2001;7:21-32. [PubMed: 12174397] 
8. Chinman M, Early D, Ebener P, et al. Getting To Outcomes: a community-based participatory approach to preventive interventions. J Interprof Care 2004;18:441-443. [PubMed: 15801559]

9. Wolff M, Maurana CA. Building effective community-academic partnerships to improve health: A qualitative study of perspectives from communities. Academic Medicine 2001;76:166-172. [PubMed: 11158838]

10. Baker EA, Homan S, Schonhoff R, et al. Principles of practice for academic/practice/community research partnerships. American Journal of Preventive Medicine 1999;16:86-93. [PubMed: 10198685]

11. Mitchell SM, Shortell SM. The governance and management of effective community health partnerships: A typology for research, policy, and practice. Milbank Quarterly 2000;78:241-289. [PubMed: 10934994]

12. Lieberman JA, Stroup TS, McEvoy JP, et al. Effectiveness of antipsychotic drugs in patients with chronic schizophrenia. New England Journal of Medicine 2005;353:1209-1223. [PubMed: 16172203] 